\title{
Combined low level light therapy and intense pulsed light therapy for the treatment of meibomian gland dysfunction
}

This article was published in the following Dove Press journal: Clinical Ophthalmology

\author{
Karl Stonecipher ${ }^{\prime}$ \\ Thomas G Abell ${ }^{2}$ \\ Bennett Chotiner ${ }^{3}$ \\ Erik Chotiner ${ }^{3}$ \\ Rick Potvin ${ }^{4}$
}

'Physicians Protocol, Greensboro, NC, USA; ${ }^{2}$ Abell Eyes, Lexington, KY, USA; ${ }^{3}$ Memorial Eye Institute, Harrisburg, PA, USA; ${ }^{4}$ Science in Vision, Akron, NY, USA

Correspondence: Karl Stonecipher Physicians Protocol, 1002 North Church Street, Suite 10I, Greensboro, NC 27408, USA

Email Stonenc@gmail.com
Purpose: To evaluate the effects of combined intense pulsed light therapy (IPL) and lowlevel light therapy (LLLT) on clinical measures of dry eye related to severe meibomian gland disease (MGD) in subjects unresponsive to previous medical management.

Patients and Methods: This was a retrospective chart review of patients treated by 4 physicians at 3 centers. All patients were documented treatment failures with traditional pharmaceutical therapy. They all had their MGD evaluated before treatment using a grading scale $(0-4)$, tear breakup time in seconds and the Ocular Surface Disease Index (OSDI) questionnaire. To be included, all patients had to have had a short course of adjunct pharmaceutical or device-related therapy, along with a combined IPL/LLLT treatment. As well, a second MGD evaluation with the same three measures had to have been conducted 1-3 months post treatment.

Results: A total of 460 eyes of 230 patients were identified for inclusion in the data set. Mean OSDI scores were significantly lower after treatment; $70.4 \%$ of patients had pretreatment OSDI scores indicative of dry eye; this dropped to $29.1 \%$ of patients after treatment. A 1-step or greater reduction in MGD grading was observed in $70 \%$ of eyes, with $28 \%$ of eyes having a 2 -step or greater reduction. Tear breakup time was $\leq 6$ seconds in $86.7 \%$ of eyes pretreatment, dropping to $33.9 \%$ of eyes after treatment. There were no ocular or facial adverse events or side effects related to the combined light treatment.

Conclusion: The use of combined IPL/LLLT for the treatment of severe MGD appears to be beneficial in patients who have failed topical and/or systemic therapy.

Keywords: LLLT, low level light therapy, IPL, intense pulsed light, meibomian gland dysfunction, ocular surface disease index

\section{Plain language summary}

Millions of people in the world suffer from dry eye disease, which can affect their work, their lifestyle and their comfort. The causes are varied, but a significant factor is the health and operation of the meibomian glands. These glands are visible just behind the eyelashes on both lids; they supply an oily "top layer" to the tears to reduce evaporation. When they are not working well, suggested treatment ranges from eye washes and warm compresses to drops and oral medications.

This study investigated the effects of light therapy for the treatment of meibomian gland issues. A combined treatment of 1) intense short pulses of light on the area of the face around the eye followed by 2) longer exposure to low level red light on the cheek and over the closed lids was administered to 230 subjects, all of whom had tried drops and oral medications in the past without success. 
Significant improvements in meibomian gland function were observed after only a single treatment. Objective and subjective measures of dry eye in subjects were also significantly reduced. There were no reported side effects on the eyes or the face. It appears that this combined light therapy may be of value in treating dry eye related to meibomian gland issues.

\section{Introduction}

Dry eye disease (DED) is a symptomatic condition characterized by tear film instability that results in inflammation and damage to the ocular surface; it is a global problem affecting hundreds of millions of people. ${ }^{1}$ It is a multifactorial disease with numerous potential initiating (and contributing) factors that result in what is often characterized as a "vicious circle" that perpetuates the DED state. ${ }^{1,2}$ Evaporative dry eye (EDE) is one of two major subtypes of dry eye, the other being aqueous deficiency dry eye; these subtypes are not mutually exclusive. In evaporative dry eye tear film evaporation increases tear osmolarity, which is a hallmark contributing factor to the inflammatory cascade associated with DED. ${ }^{2,3}$

As early as 1993, an association between increased tear evaporation and meibomian gland dysfunction (MGD) was observed. ${ }^{4}$ More recently, MGD has been recognized as a consistent risk factor for DED. ${ }^{1}$ The international Dry Eye Workshop (DEWS) II report states "Indeed, MGD, a contributor to EDE, is considered the leading cause of dry eye in clinic and population based studies". 5 This may be a function of gland dropout with age or chronic damage, or may be related to poor quality meibum, the oily substance forming the anterior lipid layer of the tear film. ${ }^{6}$ In most cases, the typical presentation is an obstruction at the opening of the meibomian gland duct, due either to keratinized cells or meibum with excessively high viscosity. Historically, treatment of MGD has ranged from warm compresses and lid scrubs to topical or systemic pharmaceutical therapy, though in recent years several new devices/procedures have been designed to promote improved outflow of meibum.

Intense pulsed light (IPL) therapy is one such procedure. It was developed based on the observation that IPL therapy for the treatment of acne rosacea appeared to lessen the severity of MGD and DED in some patients. Patients with MGD were subsequently treated using a variety of IPL protocols. A retrospective review of data from 78 patients showed that $90 \%$ had improved tear breakup time (TBUT), improved subjective lid margin appearance and better subjective meibum quality and patient satisfaction (immediately post- treatment). ${ }^{7}$ Subsequent prospective trials appear to corroborate these early findings, showing improvement in dry eye symptoms and/or meibomian gland function with IPL alone or IPL in conjunction with meibomian gland expression. ${ }^{8-11}$ The procedure has been reported to be effective up to 6 months post treatment. $^{12}$

A different kind of photobiomodulation has also been developed for dermatological and other medical uses - low level light therapy (LLLT). Athermal, atraumatic cellular photoactivation can be achieved using light emitting diodes (LEDs) of specific wavelengths. Photon interference, characteristic with LED use, results in both increased photon intensity and penetration below the skin. The photoactivation achieved is reported to result in repair to damaged or compromised cells and improved cellular function in normal cells. ${ }^{13}$ Early research in adapting this technology to MGD has demonstrated significant improvement in tear breakup time after treatment. ${ }^{14}$

A relatively new device is now CE-Marked for the treatment of MGD, which allows for the application of both the IPL and LLLT therapies described above; this is referred to in this manuscript as combined light therapy. The device is known as Eye-light ${ }^{\circledR}$ outside the USA. An equivalent unit approved for dermatological use in the USA is the Epi-C PLUS (both Espansione Marketing S.p.A., Bologna, Italy). Proprietary diagnostic equipment is used to measure the patient's level of MGD, while the user can enter the patient's Fitzpatrick skin scale. Internal software then adjusts energy levels for optimum effect. The use of gel, common with some IPL delivery systems, is not required as a patented cooling system of forced air maintains the temperature of the crystal at a non-traumatic level for the patient's skin type. In a two-step procedure, IPL is first applied to the lower periorbital area followed by LLLT over both the closed lids (upper and lower) and periorbital area; this sequence is designed to both stimulate function of the meibomian glands and to soften the meibum.

The current study was a retrospective chart review conducted to evaluate the effects of this combined light therapy (IPL and LLLT) on the function of the meibomian glands, evaluated through a subjective questionnaire, tear film breakup time and MGD severity grade as determined using a subjective scale.

\section{Patients and methods}

This retrospective chart review was approved by an institutional review board (Salus IRB, Austin, TX), which also granted a waiver of informed consent for use of patients' 
de-identified clinical data. The chart review included data from four physicians at three clinical sites. Sites treated patients for MGD with combined light therapy using the EPI-C Plus device. This involved using the IPL function of the device at a lower power than is typical for dermatological applications $\left(10-16\right.$ joules $/ \mathrm{cm}^{2}$ vs $30-46$ joules $/ \mathrm{cm}^{2}$ for dermatological use) and included subsequent LLLT. During the application of IPL patients' eyes were covered with a protective device, as recommended in the manufacturer's user manual.

Treatment for all patients consisted of two parts, IPL followed by LLLT. Patients were placed in either a prone or supine position. During the application of IPL patients' eyes were covered with a protective device, as recommended in the manufacturer's user manual. Five IPL pulses were delivered using the $12 \mathrm{~cm}^{2}$ delivery system. Three were placed along the inferior orbital rim with the device in the vertical position abutting the protective eyewear, ensuring placement to the inferior edge of the lid margin while protecting the globe. A fourth was delivered vertically behind the lateral canthus while the last one was delivered with the device horizontal along the inferior orbital rim. This sequence takes less than 5 mins for both eyes. The IPL device was then switched out for the LLLT mask, which the patient wore for 15 mins. No protective eyewear is indicated during this treatment; the mask treats only the periorbital area and the patient is instructed to keep their eyes closed, to ensure application of the LLLT fully to the upper and lower lids.

Clinical evaluations of MGD were made using several standard measures. The Ocular Surface Disease Index (OSDI) questionnaire score is a recognized standard for evaluating patient symptoms of dry eye. ${ }^{15}$ Additional evaluations included an MGD grading scale based on the quality and quantity of meibum that could be expressed and the TBUT (in seconds) measured subjectively at the slit-lamp. Endpoints were the changes in OSDI, MGD grade and TBUT from pretreatment to one to three months post treatment. In addition, all sites monitored treated patients for any treatment-related side effects.

Figure 1 shows the MGD grading scale that was used, with whole steps from 0 to 3 ; while not shown, grading in half steps was permitted. Grade 0 is normal meibum easily expressed. Grade 1 is meibum easily expressed, but with a more turbid oil. Grade 2 includes the presence of more turbid oil and a more viscous appearance, while Grade 3 is the expression of "ropy" meibum. A Grade of 4 corresponded to no expression from the glands despite multiple attempts.
The physicians involved had treated appropriate subjects who had MGD where prior pharmaceutical or device-related therapies had failed. Patients with allergies to any of the proposed treatments, or who were diagnosed with conditions which would preclude their exposure to intense pulsed light therapy or low-level light therapy were not treated. This included patients with darkly pigmented skin, as an increased risk of side effects from the application of IPL to this skin type has been noted. ${ }^{16}$ Patients treated with additional dry eye therapies were excluded from the data collection, as were pregnant or lactating females and any patient participating in any clinical study at the time of their treatment.

Patients were considered for inclusion in the dataset if they had either an Ocular Surface Disease Index (OSDI) score of 33 or higher, a TBUT of 6 seconds or less or an MGD subjective grade higher than 2 (ie 2.5 to 4 ). In addition, they had to have completed the OSDI questionnaire and had their MGD grade and TBUT subjectively determined before treatment. For consistency with the approach in one practice, patients were included only if they had been placed on a twoweek regimen of a topical gatifloxacin and prednisolone antibiotic/steroid combination three times per day in both eyes, along with oral Doxycycline - one $100 \mathrm{mg}$ tablet twice per day. This was an adjunct to the combined light therapy, but all treated patients had been previously demonstrated to be treatment failures with pharmaceutical or device therapy on single or multiple prior occasions. Finally, included patients must have had a clinic visit from one to three months after treatment, and this visit had to include an OSDI questionnaire, MGD grading and TBUT measurement.

Clinical data were tabulated in an Excel spreadsheet and then imported into an MS Access database for data checking, collation and preliminary analysis (both Microsoft Corp., Redmond, WA). Statistical analyses were performed using the Statistica data analysis software system, version 12 (TIBCO Software Inc., Palo Alto, CA, USA). Comparisons of continuous variables before and after treatment were made using a repeated measures analysis of variance (ANOVA), while categorical results were compared using the Chi-squared test or Wilcoxon signranked test. Statistical significance was set at $p=0.05$.

\section{Results}

Clinical records for a total of 230 treated patients were available for analysis. The average age was 65.5 \pm 12.0 years with a range of 28 to 95 . The majority of patients were female $(175 / 230,76 \%)$. Table 1 contains the summary data for treatment effectiveness. MGD grades 

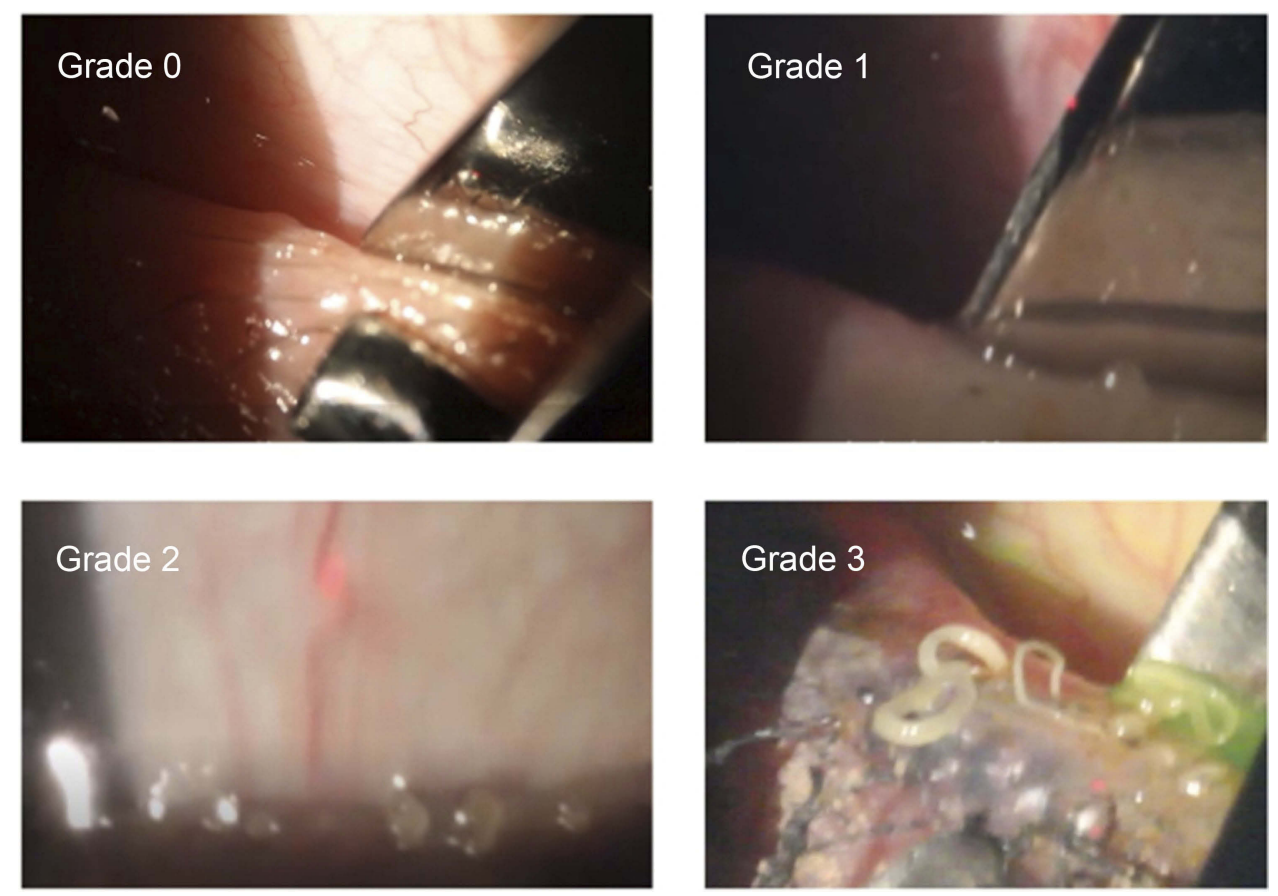

Figure I The MGD subjective grading scale.

Notes: Grade 0 is normal meibum easily expressed. Grade I is meibum easily expressed, but with with a more turbid oil. Grade 2 includes the presence of more turbid oil and a more viscous appearance. Grade 3 is the expression of "ropy" meibum. Grade 4 is no expression from the glands despite multiple attempts.

Abbreviation: MGD, meibomian gland disease.

Table I Summary statistics

\begin{tabular}{|l|c|c|c|}
\hline Measure & $\mathrm{n}$ & Pre treatment & Post treatment \\
\hline OSDI Score & 230 & $42.2 \pm 18.8(6$ to 93$)$ & $24.2 \pm 15.9$ (0 to 75$)$ \\
MGD Grade* & 460 & & 6 \\
0 & & & 34 \\
1 & & 9 & 181 \\
2 & & 175 & 179 \\
3 & & 276 & 60 \\
4 & & $4.4 \pm 2.1(0$ to 14$)$ & $8.0 \pm 3.0(1$ to I7) \\
\hline
\end{tabular}

Notes: *Intermediate scores were rounded up to the nearest full grade. Abbreviations: OSDI, Ocular Surface Disease Index; TBUT, tear breakup time; MGD, Meibomian Gland Dysfunction.

were rounded up to the nearest whole grade in this table, though half-steps were used in the analysis. Most patients $(86 \%)$ had only a single combined light therapy treatment.

Figure 2 shows the distribution of OSDI scores before and after treatment. Scores were significantly lower after treatment (Wilcoxon matched pairs test, $p<0.001$ ). Using a cutoff score of $\geq 33$ to indicate severe dry eye, $70.4 \%$ of patients (162 of 230) pretreatment and only $29.1 \%$ of subjects $(67 / 230)$ post treatment would have been classified as having severe dry eye. This difference was statistically significant (Chi-squared test, $p<0.001)$. Overall,
$71.7 \%$ of subjects $(165 / 230)$ had a decrease in their OSDI score of 10 or more points. The change in OSDI scores was not significantly different between females and males (Mann-Whitney U-test, $p=0.28$ ). There was also no correlation between the change in OSDI score and age $\left(\mathrm{r}^{2}=0.01\right)$. The change in OSDI score was similar between the three clinical sites (Kruskal-Wallis test, $p=0.35$ ).

The MGD grade was also significantly lower after treatment (Wilcoxon matched pairs test, $p<0.001$ ). No eyes had an MGD grade of 2 or lower before treatment; after treatment, 39.3\% of eyes (181/460) had an MGD grade of 2 or lower. This was a statistically significant difference (Chisquared test, $p<0.001)$. An MGD grade reduction of 1 or more was observed in $69.6 \%$ of eyes $(320 / 460)$ while $28.0 \%$ of eyes (129/460) had a grade reduction of 2 or more. The change in MGD grade was not different between the sexes (Mann-Whitney U-test, $p=0.43$ ).

Figure 3 shows the distribution of TBUT before and after treatment. The mean breakup time nearly doubled after treatment; this change was statistically significant (4.4-8.0 seconds, ANOVA, $p<0.01$ ). Pretreatment, 86.7\% of eyes (399/460) had a TBUT $\leq 6$ seconds, suggestive of dry eye. Post treatment, this dropped to $33.9 \%$ (156/460). This change was statistically significant (Chi-squared test, 


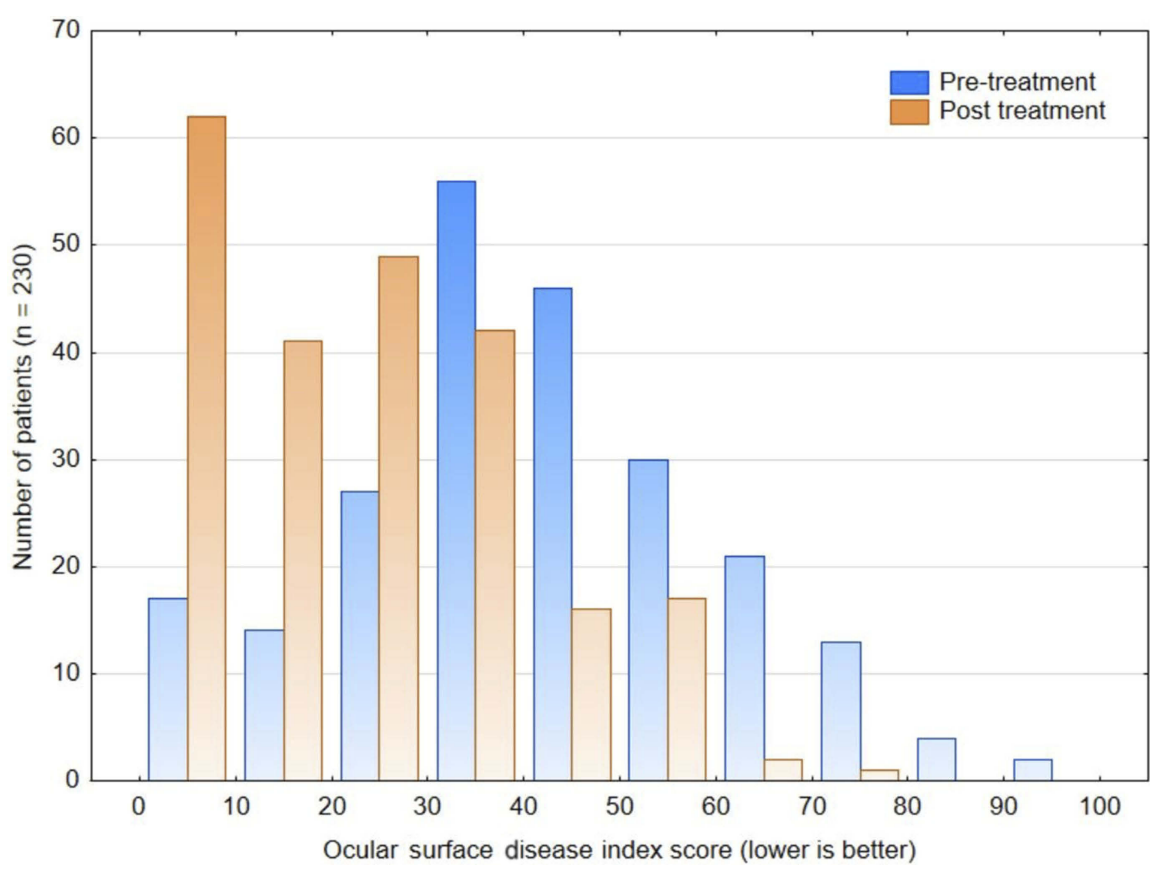

Figure 2 The distribution of ocular surface disease index scores before and after treatment.

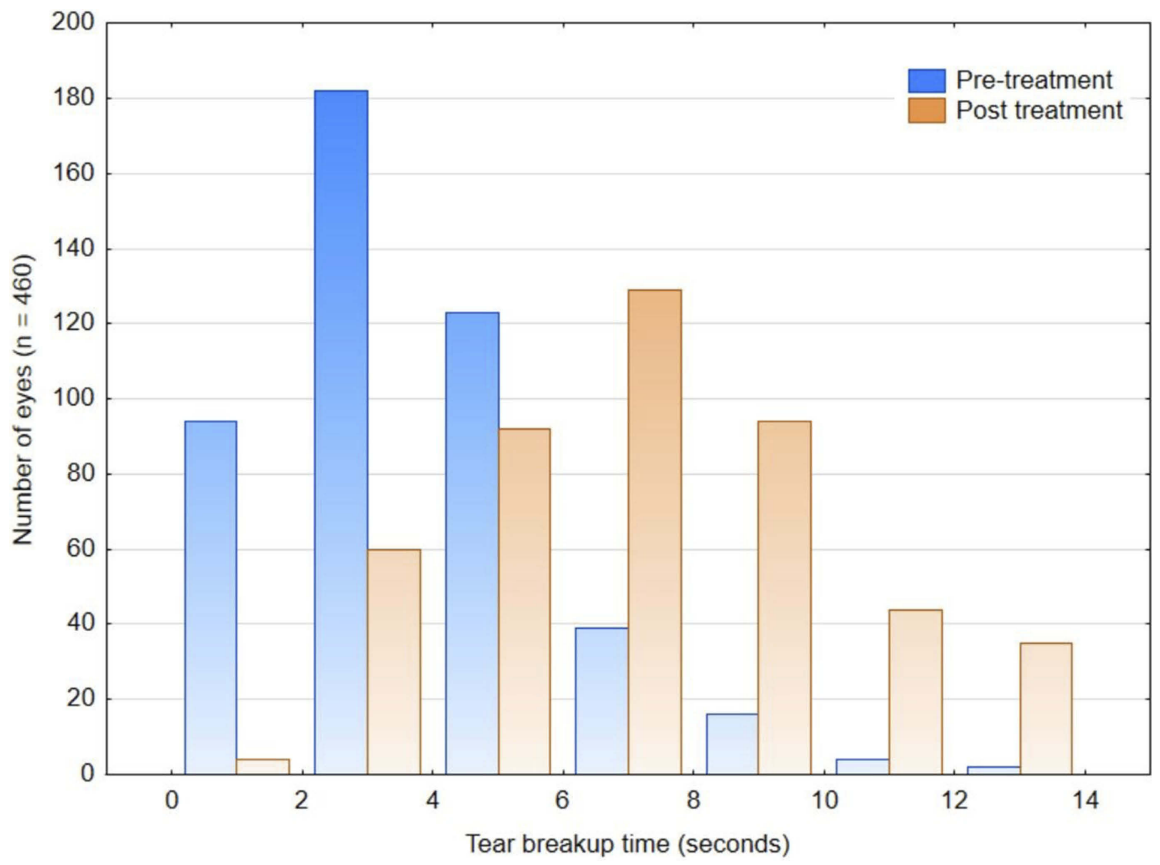

Figure 3 The distribution of tear breakup time before and after treatment.

$p<0.001)$. TBUT increased by 3 seconds or more in $60 \%$ of eyes (277/460). The change in TBUT was significantly different between the sexes $(3.4 \pm 3.2$ seconds for females, $4.1 \pm 2.8$ seconds for males, ANOVA, $p=0.02$ ); this difference was not considered clinically significant. There was no association between the change in TBUT and age $\left(r^{2}=0.01\right)$.

In the 460 eyes treated here, there were no reported facial or ocular side effects or adverse events reported by any of the physicians. 


\section{Discussion}

To our knowledge, this is the first published study to investigate the effects of a combined IPL and LLLT therapy for the treatment of MGD. The current study demonstrated significant improvement in the severity of MGD in most patients when the combined IPL/LLLT therapy was applied in a single treatment. The essentially thermalbased effects of IPL treatment are presumably augmented by the additional photobiomodulation effects of the LLLT treatment on the lids and periorbital area. The application of LLLT to the upper lids, an area not typically treated with IPL therapy alone, may be an additional contributing factor to the improvement in MGD observed.

Comparisons with other studies are difficult because of differences in the methodologies used to measure changes to subjective and objective signs and symptoms. In addition, treatment protocols vary widely. However, the improvements reported here are similar to those reported with IPL in previous studies. The average TBUT at 1-3 months posttreatment was slightly higher than was reported by Arita et al in a study examining IPL only with meibomian gland expression at 24 and 32 weeks after treatment. ${ }^{10}$ Improvements in OSDI and TBUT appear consistent with those achieved by Albietz et $\mathrm{al}^{15}$ and somewhat better than those reported by Gupta et al. ${ }^{17}$ This is despite the fact that most patients had only a single treatment, where the number of treatments for IPL-only therapy in the studies above was often 3 to 5. Unlike the treatments in these other studies, the current treatment eliminated the need for a gel interface; this is a possible advantage for both patients and physicians.

The presumed mechanism of action of IPL is essentially thermal. Research has demonstrated the absorption of light energy by hemoglobin, creating heat that can destroy small telangiectatic blood vessels; ${ }^{18}$ this may reduce the influx of inflammatory mediators to the eyelid and meibomian glands. Other reported effects include: a) raising the temperature of the blood in larger vessels of the lid, which may be sufficient to heat the meibum in clogged glands up to its phase-transition temperature, allowing it to flow more freely, b) eradication of Demodex folliculum mites on the lids, with a corresponding drop in the microbial load (and potential clogging debris) at the meibomian gland orifices and c) inhibiting the secretion of pro-inflammatory cytokines and promoting secretion of anti-inflammatory cytokines near the lids and meibomian glands. ${ }^{19}$ In contrast, LLLT is believe to be athermal, with photoactivation the presumed mechanism. ${ }^{13}$
There are limitations to the current study. The diagnostic evaluation tool recommended for use with the combined light therapy device was not available, which meant that subjective measurements for MGD grading were required. This also meant that the therapy could not be optimized on an individual eye basis; consequently, results here may be somewhat conservative. Use of a prophylactic pharmaceutical regimen before combined treatment may also have introduced a confounding factor, though all presenting subjects had failed to improve with similar treatments in the past. In addition, there was no means to separate the individual effects of the combined therapy. Future studies are planned that will address these concerns. For instance, a future randomized prospective study comparing the application of IPL only and sham LLLT to the combined IPL/LLLT therapy is under consideration, with no concurrent pharmaceutical use; post treatment evaluations will be performed by individuals masked to the treatment plan. While not studied here, it is also likely that treatment with combined light therapy would be effective in a wider range of eyes than just those with severe or unresponsive MGD; a study including subjects with different levels of MGD would be helpful in to demonstrate this.

\section{Conclusion}

Combined IPL and LLLT therapies in a single treatment produced significant improvements in tear breakup time and MGD grading scores, with an associated improvement in the patient's subjective OSDI score, one to three months after treatment.

\section{Acknowledgments}

Physicians Protocol (KS) provided funding to Science in Vision to assist with data analysis and preparation of this manuscript.

\section{Disclosure}

Dr Karl Stonecipher is a consultant to Espansione Marketing S.p.A., Alcon, Allergan, and Bausch Health. $\mathrm{He}$ reports grants and personal fees from Allergan, Alcon, Espansione, and Bausch, during the conduct of the study. He also received grants and personal fees from Allergan, Alcon, Bausch, and Espansione, outside the submitted work. Dr Rick Potvin reports personal fees from Physicians Protocol, during the conduct of the study. The authors report no other conflicts of interest in this work. 


\section{References}

1. Craig JP, Nelson JD, Azar DT, et al. TFOS DEWS II report executive summary. Ocul Surf. 2017;15(4):802-812. Epub 2017 Aug 8 Review. PubMed PMID: 28797892. doi:10.1016/j.jtos.2017.08.003

2. Baudouin C, Messmer EM, Aragona P, et al. Revisiting the vicious circle of dry eye disease: a focus on the pathophysiology of meibomian gland dysfunction. $\mathrm{Br} J$ Ophthalmol. 2016;100(3):300-306. Epub 2016 Jan 18. Review. PubMed PMID: 26781133; PubMed Central PMCID: PMC4789719. doi:10.1136/bjophthalmol-2015307415

3. Nichols KK, Foulks GN, Bron AJ, et al. The international workshop on meibomian gland dysfunction: executive summary. Invest Ophthalmol Vis Sci. 2011;52(4):1922-1929. Review. PubMed PMID: 21450913; PubMed Central PMCID: PMC3072157. doi:10.1167/iovs.10-6997a

4. Mathers WD. Ocular evaporation in meibomian gland dysfunction and dry eye. Ophthalmology. 1993;100(3):347-351.

5. Nichols JJ, Tsubota K, Stapleton F. TFOS DEWS II definition and classification report. Ocul Surf. 2017;15(3):276-283. Epub 2017 Jul 20. Review. PubMed PMID: 28736335. doi:10.1016/j.jtos.2017.05.008

6. Nebbioso M, Del Regno P, Gharbiya M, Sacchetti M, Plateroti R, Lambiase A. Analysis of the pathogenic factors and management of dry eye in ocular surface disorders. Int J Mol Sci. 2017;18(8): pii: E1764.

7. Toyos R, McGill W, Briscoe D. Intense pulsed light treatment for dry eye disease due to meibomian gland dysfunction; a 3-year retrospective study. Photomed Laser Surg. 2015;33(1):41-46. PubMed PMID: 25594770; PubMed Central PMCID: PMC4298157. doi:10.1089/pho.2014.3819

8. Craig JP, Chen YH, Turnbull PR. Prospective trial of intense pulsed light for the treatment of meibomian gland dysfunction. Invest Ophthalmol Vis Sci. 2015;56(3):1965-1970. PubMed PMID: 25678687. doi:10.1167/iovs.14-15764

9. Yin Y, Liu N, Gong L, Song N. Changes in the meibomian gland after exposure to intense pulsed light in meibomian gland dysfunction (MGD) patients. Curr Eye Res. 2018;43(3):308-313. doi:10.1080/ 02713683.2017.1406525

10. Arita R, Fukuoka S, Morishige N. Therapeutic efficacy of intense pulsed light in patients with refractory meibomian gland dysfunction Ocul Surf. 2019;17(1):104-110. Epub 2018 Nov 13. PubMed PMID: 30445177. doi:10.1016/j.jtos.2018.11.004
11. Dell SJ, Gaster RN, Barbarino SC, Cunningham DN. Prospective evaluation of intense pulsed light and meibomian gland expression efficacy on relieving signs and symptoms of dry eye disease due to meibomian gland dysfunction. Clin Ophthalmol. 2017;11:817-827. eCollection 2017. PubMed PMID: 28496300; PubMed Central PMCID: PMC5422561. doi:10.2147/OPTH.S130706

12. Rong B, Tang Y, Liu R, et al. Long-term effects of intense pulsed light combined with meibomian gland expression in the treatment of meibomian gland dysfunction. Photomed Laser Surg. 2018;36 (10):562-567. Epub 2018 Sep 22. PubMed PMID: 30251914 doi: $10.1089 /$ pho. 2018.4499

13. Kim WS, Calderhead RG. Is light-emitting diode phototherapy (LED-LLLT) really effective? Laser Ther. 2011;20(3):205-215. Review. PubMed PMID: 24155530; PubMed Central PMCID: PMC3799034.

14. Toyos R, Briscoe D, Toyos M. The effects of red light technology on dry eye disease due to meibomian gland dysfunction. JOJ Ophthal. 2017;3(5):555624.

15. Schiffman RM, Christianson MD, Jacobsen G, Hirsch JD, Reis BL. Reliability and validity of the ocular surface disease index. Arch Ophthalmol. 2000;118(5):615-621.

16. Thaysen-Petersen D, Erlendsson AM, Nash JF, et al. Side effects from intense pulsed light: importance of skin pigmentation, fluence level and ultraviolet radiation-A randomized controlled trial. Lasers Surg Med. 2017;49(1):88-96. doi:10.1002/lsm.22566

17. Gupta PK, Vora GK, Matossian C, Kim M, Stinnett S. Outcomes of intense pulsed light therapy for treatment of evaporative dry eye disease. Can J Ophthalmol. 2016;51(4):249-253. Epub 2016 Jun 22. PubMed PMID: 27521662. doi:10.1016/j.jcjo.2016.01.005

18. Papageorgiou P, Clayton W, Norwood S, Chopra S, Rustin M. Treatment of rosacea with intense pulsed light: significant improvement and long-lasting results. Br J Dermatol. 2008;159(3):628-632. doi:10.1111/j.1365-2133.2008.08702.x

19. Dell SJ. Intense pulsed light for evaporative dry eye disease. Clin Ophthalmol. 2017;11:1167-1173. eCollection 2017. Review. PubMed PMID: 28790801. doi:10.2147/OPTH.S139894
Clinical Ophthalmology

\section{Publish your work in this journal}

Clinical Ophthalmology is an international, peer-reviewed journal covering all subspecialties within ophthalmology. Key topics include: Optometry; Visual science; Pharmacology and drug therapy in eye diseases; Basic Sciences; Primary and Secondary eye care; Patient Safety and Quality of Care Improvements. This journal is indexed on PubMed

\section{Dovepress}

Central and CAS, and is the official journal of The Society of Clinical Ophthalmology (SCO). The manuscript management system is completely online and includes a very quick and fair peer-review system, which is all easy to use. Visit http://www.dovepress.com/ testimonials.php to read real quotes from published authors. 